\title{
Making Energy System Models Useful: Good Practice in the Modelling of Multiple Vectors
}

Graeme S Hawker graeme.hawker@strath.ac.uk, Keith R W Bell

Department of Electronic and Electrical Engineering, University of Strathclyde, Glasgow, UK

\begin{abstract}
Energy system models which cover multiple vectors have become increasingly used to provide an evidence base for policy and commercial decisions in real-world energy systems undergoing change. In particular, such models are often used to derive 'optimal' pathways to decarbonisation considering the planning or operation of systems with multiple technology options. This paper explores how the concept of 'usefulness' - the applicability and relevance of modelling outcomes - may be used to establish criteria for modelling design and practice at the outset, and looks at the difficulties that may be faced in achieving this. The application should inform the choice of modelling framework and the manner in which tractability should be addressed and results meaningfully presented. A process of continuous engagement is proposed which guides modelling work towards 'useful' outcomes, as well as mitigating the danger of results being more reflective of design choices than the properties of the real-world systems being modelled. Because of the difficulties in maintaining and auditing complex datasets spanning expertise from multiple sectors, there is a clear role for independent data curators to facilitate rigour in model parameterisation and to allow consistency between modelling efforts. Specialists from the different disciplines represented should be engaged to ensure that data have been interpreted and applied correctly. All modelling choices should be clearly documented along with advice on their possible implications in respect of use of the results.
\end{abstract}

\section{INTRODUCTION}

In the construction of an energy system model, the modeller has a broad set of decisions to make, among others: the choice of framework, which constraints to apply, what input data to source and which scenarios to evaluate. Often modellers may make use of pre-existing tools, devised for a specific purpose, which may not be wholly consistent with the modeller's new goal. In this paper we evaluate the context within which energy system models - and specifically models which analyse multiple vectors together - are used in order to propose a set of criteria and design choices which a modeller might use to inform the first and subsequent steps of their activity. In doing so, we establish the concept of 'usefulness' in such modelling activity, and how the process of ensuring that the outputs of modelling activity are 'useful' to others might be enacted throughout the research and development process.

In recent years there has been significant growth in the use of models which do not analyse a particular form of energy in isolation, but which attempt to simulate or optimise multiple forms of energy (often termed vectors) across an energy system, defined as "the combined processes of acquiring and using energy in a given society or economy" (Jaccard, 2006). An energy system may include a number of processes, such as resource extraction, refining, transportation, storage and conversion to end-use energy services (Pfenninger, Hawkes, \& Keirstead, 2014). An optimisation of system design or operation must take account of the balance between supply and demand and the choices available in respect of each. The energy system involves social and technical concerns, 
comprising not only fuels, conversion equipment and pipelines but also markets, consumers and institutions, each of which may have a distinct local realisation. At a policy level, governments and national institutions have a deep interest in the whole system view of energy, which seeks to capture both the resourcing and flow of energy across an entire nation and sometimes beyond.

If a policymaker seeks to fulfil a quantitative goal (for example, a particular level of emissions abatement), then the use of quantitative modelling becomes key to capturing and understanding the current-day flows of energy within a system which may represent one of a number of different scales from local to national or international. From there the policymaker may project forwards to estimate the required levels of intervention (as well as the necessary policy structures) that will be required to meet a specific future target (Mancarella, Andersson, Peças-Lopes, \& Bell, 2016). This permits policies such as carbon taxation, technology subsidies, or behavioural interventions, to be tested against a known system background, and so can be used to build up an evidence base for the implementation of new legislation. This further addresses the challenge of Energy Systems Integration (ESI) - the process of coordinating and planning energy systems across multiple energy vectors and geographical scales to deliver cost-effective energy services while minimising environmental impacts and considering interactions with other large-scale infrastructures such as water and transport (O'Malley et al., 2016).

Figure 1 illustrates the key stages in the energy modelling process, both in terms of characterising and defining the parameters and variables endogenous to the model, and the scenarios and exogenous parameters relating to the context within which the model is operated.

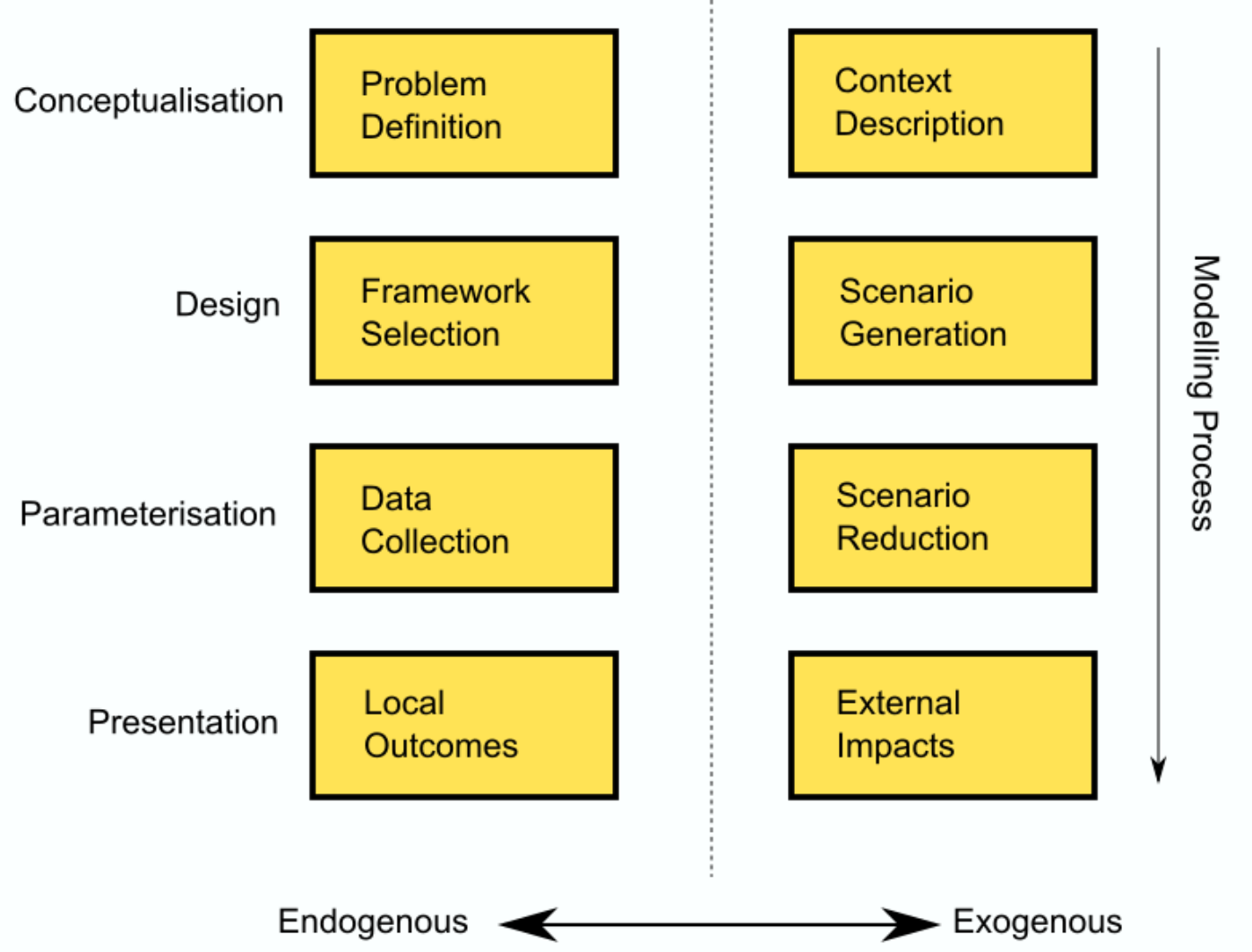

Figure 1 - An overview of the modelling stages, separating properties internal to the model from its external context 
This paper examines the qualities of an energy system model that are necessary to make its output useful and worthwhile. It then assesses the different general forms of energy system models that are currently in use and how these criteria may be applied to them. The processes involved in applying the outputs of multi-vector energy system models to the real world (in policymaking or otherwise) are discussed, which in turn is used to define a set of recommendations for future model design, use and maintenance.

\section{DEFINING A ‘USEFUL’ ENERGY MODEL}

The frequently-cited aphorism "all models are wrong but some are useful" (Box, 1979) highlights the key motivating drive for models to be illuminating and insightful despite their necessary shortcomings and simplifications. This in turn creates the need to assess how modelling created to date has ensured that findings are applicable and valuable. Where applicability and value have been questionable, what has caused it?

Within the context of multi-vector and whole system energy models, the authors here define 'usefulness' as fulfilling at least one of the following criteria.

- Quantification: The model quantitatively confirms or rejects the hypothesised contribution that can be made by a particular technology/policy, set of technologies/policies or a particular implementation of those technologies/policies towards environmental concerns, cost, resilience or security.

- Conceptualisation: The model demonstrates (qualitatively or quantitatively) a previously unknown contribution.

- Comparison: The model robustly compares and contrasts the contributions of different technologies, policies and/or implementations, such that the results give supporting evidence to a decision which must weigh up multiple possible options.

- Contextualisation: The model adds additional detail to previous analyses on how technologies/policies might be implemented and how they may interact with complex extant systems.

- Certainty: The model reduces the uncertainty (and by extension the risk) associated with one of the above.

- Application: The model illustrates some aspect of the above within a specific real-world context.

A specific model comprises a mathematical framework - a set of equations, perhaps only algebraic, but perhaps also differential or stochastic - designed for a particular analytical purpose, and the parameterisation of that set of equations representing - to some degree of accuracy - a specific realworld case. The usefulness of a modelling framework can be enhanced through the ease by which a given set of equations can be solved for a different set of parameters and inputs representing a different real-world case. It is not necessary, however, for all modelling to attempt to investigate realworld cases. Modelling conducted purely for the purposes of abstract insight may also provide value to inform further research, and is often the first exploratory step in seeking to evaluate a new area of analysis. This is, in effect, 'prototyping' of modelling that may initially explore the above criteria and subsequently inform modelling of the real-world cases, or may indicate unfruitful avenues of investigation prior to the investment of large amounts of modelling resource.

An additional dimension to model usefulness is that of timeliness - the model should seek to provide evidence that is useful for current or developing narratives. If the results of the modelling are intended 
to provide evidence to a policymaker, then further consideration should be made of whether it is actionable - do the results describe or support interventions that can be made by system actors? Are these interventions achievable not only now, but also in alternative future visions of the system? This particular aspect is developed further in the discussion on policymaking below.

\section{MODEL CHOICE AND INTENDED APPLICATION}

In order to ensure that the above criteria are met, it is key that the choice of modelling framework is appropriate for the intended application. In this section we provide a high-level summary of the main types of multi-vector energy model ${ }^{1}$, with a view towards understanding how they might contribute to different applications of results, and the separation between modelling frameworks and scenario selection.

\section{Overview of model types}

Historically, the vast majority of energy-related models have concerned a single vector or carrier such as electricity or gas, and are restricted in scope to a system specifically delimited, for example modelling the economically optimal dispatch of electricity in a power system or the secure provision of gas storage capacity across a transmission network. In contrast, a multi-vector or multi-carrier energy system model concerns energy supplied, consumed and transported across multiple vectors or carriers, such as the joint optimal dispatch of electricity and gas. A whole system energy model not only extends analysis across multiple vectors, but also seeks to characterise and analyse the full range of energy flows - from raw resources through to end consumer demands - for a given spatial area over a given timescale, normally incorporating key metrics such as costs and/or emissions associated with meeting demand.

A distinction may be made in the primary analytical approach taken by an energy model (Beeck, 1999). Top-down energy system models look at behavioural realism within a macro-economic framework, with exogenously generated variables representing abstractions of the aggregate properties of the energy system. The analysis seeks to investigate economy-wide responses to policies, such as through changes to income, GDP or competitiveness. For example, the UKENVI model (Allan, Hanley, McGregor, Swales, \& Turner, 2007) is a Computable General Equilibrium (CGE) model of the UK energy system, economy and environment which is used to investigate the response of the UK economy to improvements in industrial efficiency. Bottom-up models, conversely, are technologically explicit and usually include cost and emissions definitions. They may be used to explore technical opportunities and trade-offs between solutions and sectors, as typified by the TIMES modelling framework (ETSAP, 2008).

As an example, in a bottom-up model, energy demand might be equal to the sum of the number of people demanding a particular energy service at a point in time multiplied by the energy consumption associated with each service, with both parameters exogenous to the model. This aggregated energy demand then drives the derivation of a mixture of supply-side technologies capable of meeting any formalised constraints around that demanded volume of energy. Generally, bottom-up models are seen as optimistic (as they assume there are no behavioural barriers to the uptake of technologies),

\footnotetext{
${ }^{1}$ For a more comprehensive literature review of energy models, see for example (Müller, Gardumi, \& Hülk, 2018), (Mancarella et al., 2016) and (Hall \& Buckley, 2016)
} 
whereas top-down models are often pessimistic, over-estimating the cost of future technologies (van Vuuren et al., 2009).

Alternatively, 'hybrid' models may exist between the two and incorporate technological detail into a macroeconomic framework, either by soft-linking top-down and bottom-up models, or by hardlinking into a single integrated model. For example, the Long-range Energy Alternatives Planning system (LEAP) (Heaps, 2016) integrates a top-down behavioural demand model with a bottom-up energy supply model. A model - such as PRIMES (E3Mlab of ICCS/NTUA, 2008) - may also be modular, with different modelling approaches used in a selection of sub-models which are appropriately inter-linked.

\section{Model Purpose}

A model may be constructed for the purposes of forecasting - attempting to predict future patterns of energy transfer, consumption or behaviours in response to projected changes in exogenous factors. Instead, a model may be used for hindcasting - historical patterns are reconstructed as closely as possible, using a deterministic model, in order to identify underlying causes and mechanisms. Cambridge Econometrics' MDM-E3, for example, is an energy-environment-economy (E3) model which can be used to forecast changes in economic structure, energy demand and resulting environmental emissions (Allan, Gilmartin, McGregor, Swales, \& Turner, 2009), based on feedback effects from different sectors, in response to energy prices or other stimuli.

Similarly, a model may be constructed for exploring energy futures - to determine the potential changes in energy services and provision under different policy options, constraints or pricing. For example, the Department of Energy and Climate Change (DECC) 2050 Calculator (Department of Energy and Climate Change, 2011) allowed members of the public to engage with UK energy policymaking and derive their own UK energy futures based on a range of policy and technology options to meet emissions reduction targets. These futures may include analysis of the specific energy demand from different sources to enable different economic activities, or may investigate the impacts of external energy supply and imports/exports.

An optimization model will seek to either maximise or minimise an 'objective function' - often finding the least cost solution - subject to a set of constraints (such as defined by atmospheric emissions, energy security or reliability). There are a number of mathematical approaches within the field of optimisation. Typical formulations might involve each variable taking a continuous monotonic form, either linear or non-linear, or may incorporate more complex mixed-integer variable sets where individual elements may be turned 'off' or 'on' (such as when deciding whether to invest in individual large elements of infrastructure). Various approaches such as dynamic programming (e.g. as applied to the problem of local energy system management in (Tashiro, Tamura, \& Yasuda, 2011)) allow the exploration of a greater solution space through disaggregation of the system into multiple, coupled sub problems. Figure 2 illustrates the output of a local-scale optimisation model across multiple energy vectors, meeting domestic heat demand subject to network constraints through the least-emissions dispatch of thermal storage.

In a simulation model $\mathrm{l}^{2}$, the operation of an existing pre-defined configuration of an energy system is modelled either for a single snapshot or in a time-sequential manner, in order to determine the actual

\footnotetext{
${ }^{2}$ We here refer to simulation as a subset of optimisation, as opposed to the usage of the term within control theory to define local algorithms operating to keep a system within specified conditions.
} 
dispatch and flows of energy for that system. Typical outputs will be the utilisation rates of devices and implied costs. This might be used, for example, to determine if a given configuration (perhaps defined as the optimal investment from a separate model) leads to a secure and operable system when investigated at shorter time horizons. For example, in (Chiodi, Deane, Gargiulo, \& Ó'Gallachóir, 2011) the Irish Single Electricity Market is simulated for multiple runs over one year in order to validate results within the wider Irish TIMES model. In many cases, what is intended to be simulated involves some level of decision making by actors in the system. Often, these are represented by an optimisation that acts as a proxy for the decision making process within a simulation (Karangelos \& Wehenkel, 2016).

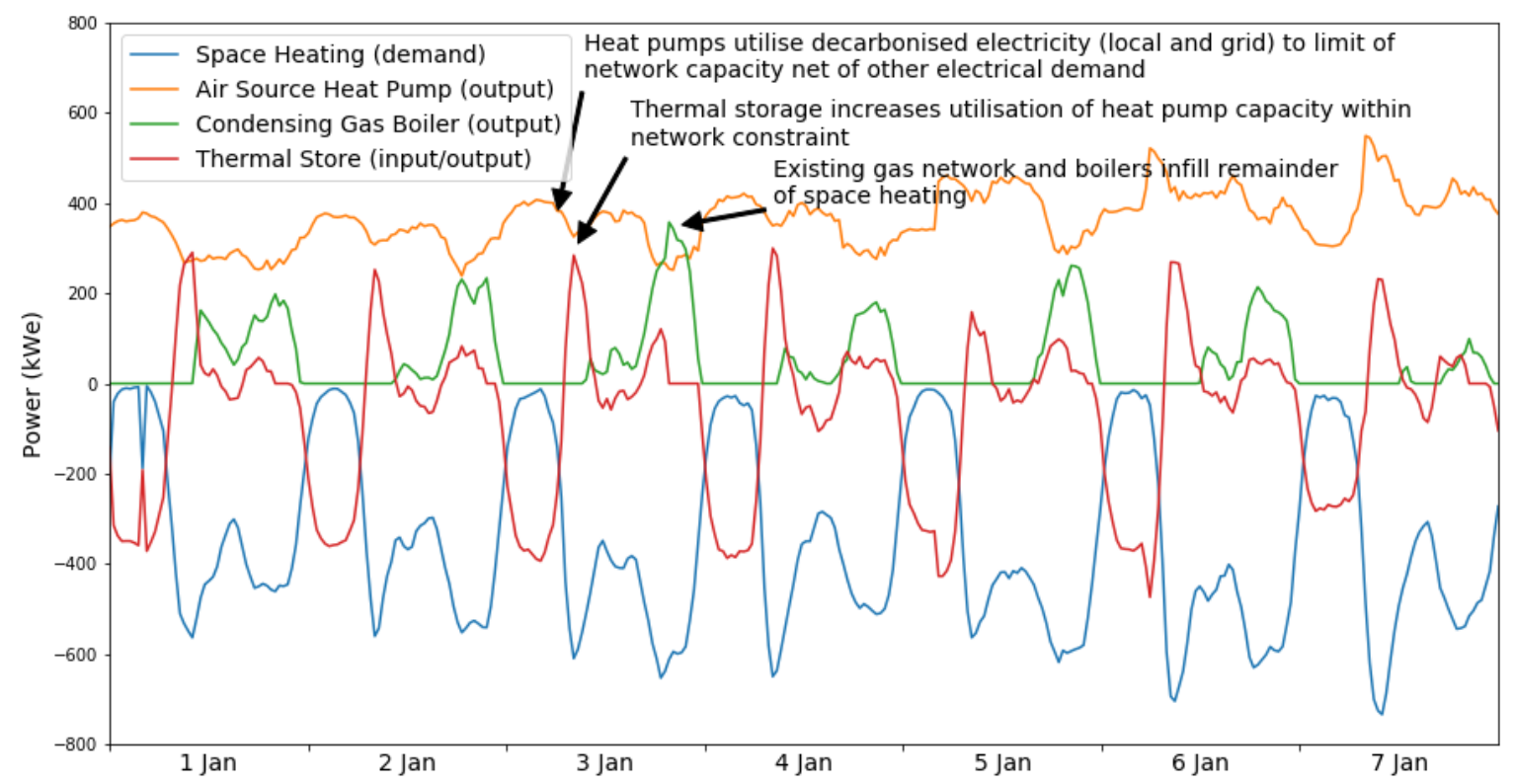

Figure 2 - example output from an optimisation-based energy simulation model, dispatching multiple energy vectors to supply domestic heat demand with the goal of emissions minimisation while respecting network limits. Adapted from (Hawker, 2018)

Within most of the above-described methodologies, stochastic variants may be created which aim to describe the impacts of variations in the exogenous variables, such as external energy prices or scenarios around the availability of renewable resources. This variation may either be captured endogenously through formulations which include stochastic optimisation, such as in (Wickart \& Madlener, 2007), or reductive methods such as Monte-Carlo simulation may be used which involve multiple model runs with exogenous variables sampled from appropriately-constructed random distributions, such as in (Zhou et al., 2013). This sampling methodology may also be used to disaggregate input datasets (such as to determine the contribution from a renewable technology at a specific point in time given an average contribution over a sampled period). In most cases, inclusion of stochasticity within an energy model greatly increases the level of computational complexity.

Within the above classifications, many models will use cost as their main determinant, either as a means of defining the objective function within an optimisation, or to calculate the cost of a particular scenario undergoing simulation. Costs may be exogenous, as in fuel prices, or generated endogenously through market simulation using supply curves generated from technology parameters. Technologies may be represented either through a single levelised cost, or in detail with capital/fixed costs, fixed operational costs and variable operational costs. Transactions (such as transmission of a 
fuel commodity) may also have associated costs. Lastly, emissions may either be governed by a hard constraint, or simply through the application of a cost per emissions (Mancarella et al., 2016). Models such as TIMES (ETSAP, 2008) allow the calculation of implied carbon costs based on emissions constraints.

Agent based methods recognise, instead, that a traditional optimization model implies the presence of a strategic planner who does not exist in reality, and instead the uptake and utilisation of new technologies is governed by decisions made by individual system actors making autonomous decisions. This area of investigation chooses to model these decisions independently by characterising, for example, the utility functions of each system actor and representing them as acting in a more myopic sense than an 'all-knowing' strategic planner. Agent-based modelling is commonly used to study interactions among multiple decision entities (Ma \& Nakamori, 2009). Typically, this assists in determining whether externally optimal decisions will be supported by real-world participants, and to investigate whether proposed policies and incentives are likely to generate a desired set of behaviours that entail uptake of desired technologies. For example, in (Stephan \& Sullivan, 2004) the interactions between actors in transport and fuel delivery are used to evaluate the potential transition to hydrogen-based transport networks.

\section{Scenario Selection}

While the above determines the choice of modelling framework, it is also key to consider the scenarios that should be investigated within that framework. Modelling frameworks and scenarios are not necessarily mutually exclusive; a modeller making a choice about the parameters of their model or the initial conditions is, in effect, choosing a scenario. When modelling the future, those choices should be acknowledged and based on a clear narrative. The model is, then, adding detail to that scenario, quantifying aspects of the system that it may otherwise be difficult to assess. If a model is being used to simulate a social system or socio-technical system (such as "the economy" or "the energy system") or to choose certain things to achieve a particular outcome in a socio-technical system, it implicitly represents social processes within that system, e.g. how consumers respond to prices or other signals.

For most practical problems, the wealth of potential scenarios will be too large (Growe-Kuska, Heitsch, \& Romisch, 2003), and some form of scenario reduction must be considered. This raises a danger of the modeller prejudicing results purely through introducing bias into this process. In the next section we discuss the involvement of stakeholders in the scenario selection process to maintain relevance and objectivity. An alternative approach is to probabilistically weight scenarios and/or to create scenario trees reflecting future evolutionary paths of the system under study (Epe et al., 2009).

Within the consideration of the 'timeliness' and relevance of a modelling effort, there is also a tendency for scenario choice to reflect contemporary debates (Trutnevyte, McDowall, Tomei, \& Keppo, 2016), meaning that model results may have a 'use-by' date beyond which the real-world systems have diverged significantly from the scenario assumptions made. This inconstant context also presents difficulties in extrapolating from known extant systems to future scenarios, such as extending a model from the understood behaviour of a single electric vehicle (EV) connecting to a distribution network, to a case with the majority of vehicles being electrically powered. Cases such as this, where technologies move from edge cases to high utilisation rates, may mean that new factors need to be considered which were not previously within scope. In the EV case, the includes electricity network impacts such as falls in voltages and breach of network thermal limits. 
Figure 3 illustrates the relationship between scenarios representing either parameters that are exogenous to the model - different futures - or those that are endogenous to it and created within it. In this case, given sets of generation capacities, operating costs and underlying patterns of demand for electricity - yearly 'macro scenarios' postulated via a separate process that takes account of scenarios describing government policies and conditions within the wider economy - were evaluated in terms of their impact on hourly operation of the system, assessed for a range of 'micro-scenarios' generated within the modelling framework via sampling of availability of generation, variation of demand and short-term variations of prices. This indicates that scenario selection and reduction can be treated hierarchically to represent different endogenous and exogenous elements at appropriate temporal and spatial resolutions.

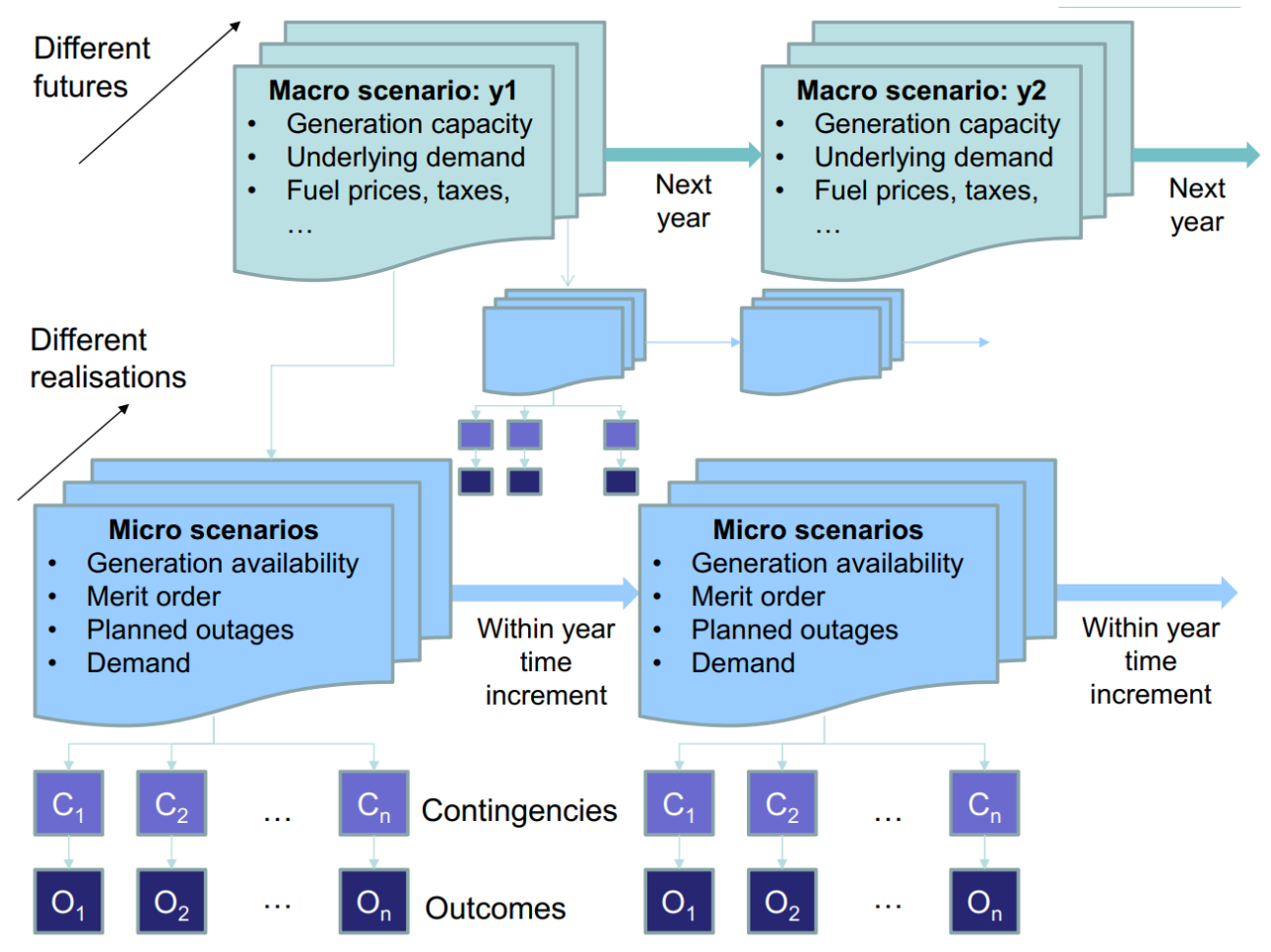

Figure 3 - The relationship between scenarios as exogenous visions of the future and scenarios as different realisations of local variables, shown here for the example of investment in generation capacity from (Bukhsh, Bell, Vergnol, Weynants, \& Sprooten, 2018).

\section{APPLICATION OF MODEL RESULTS}

The above discussion illustrates the need for the intended use of a model's results to be considered during the process of formulation. However, many models may take many years to develop, and neither the eventual application nor the sources of data that may become available may be known at the outset. This means that there is a benefit to considering the co-evolution of policy with modelling capability.

\section{Generation of Energy Policy from Energy System Modelling}

The effects of different policy interventions, such as taxation, subsidy or the imposition of minimum technical standards, can be explored across multiple linked sectors (such as transport, agriculture, housing and others) in order to understand trade-offs and inter-relationships between areas of the 
economy which may not be evident from analysis of single carriers or discrete subsets of energy use. In this respect, the use of a cross-sectoral energy system model - i.e. one containing representation of all sectors relevant to energy production and consumption - which characterises the totality of energy demand and supply, across a nation or continent, allows a government to determine if a particular policy mix will reach a desired target such as total carbon abatement. Given their limitations, such models should not be relied on to give the end answer in terms of the idealised technology and policy mix; instead, modelling should be used to deliver insights - a model might not succeed in answering a particular question but can help to reveal a next set of questions. Whole system and individual sector models allow an assessment of costs, trade-offs, possible pathways and sensitivities related to achieving emission targets within the constraints of energy security against a background of uncertainty in both the quality of a model's representation of the physical behaviour of a system - the set of equations solved - and in the values of parameters (Strachan, 2011). This process is generally iterative, both in terms of the gradual improvement of the central models used in the policy-making process (such as MARKAL within the development of the UK Energy White Papers in 2003 and 2007) but also in the incorporation of sectoral and macro-economic models into holistic analyses.

It is, indeed, the role of multi-vector models to ensure that a holistic view of the trade-off between sectors can be assessed in a manner that cannot be achieved solely through the separate analyses of each sector. For example, a recent UK government plan on reducing nitrogen dioxide emissions from vehicles acknowledges the close link between carbon emission reduction and desirable air quality improvements, despite having separate causative emissions and distinct scales of impact (DEFRA \& Department for Transport (DfT), 2017).

Mirakyan \& De Guio (2013) posit that there are 4 key stages to policy setting within energy systems, summarised in Figure 4. This illustrates the requirement for early inclusion of stakeholders in order to direct both model design and scenario selection, as well as soliciting their responses to modelling results in order to drive assessment and policy determination.

\begin{tabular}{|c|c|}
\hline $\begin{array}{l}\text { 1: Preparation / Orientation } \\
\text { - Rough analysis of situation } \\
\text { - Formulate Questions / Targets } \\
\text { - Question stakeholders }\end{array}$ & $\begin{array}{l}\text { 2: Model Design / Analysis } \\
\text { - Establish formal tools } \\
\text { - Derive scenarios } \\
\text { - } \text { Conduct modelling }\end{array}$ \\
\hline $\begin{array}{l}\text { 4: Implementation / Monitoring } \\
\text { - Enact policies } \\
\text { - Assess success } \\
\text { - Inform successive rounds }\end{array}$ & $\begin{array}{l}\text { 3: Prioritisation / Decision } \\
\text { - Review by stakeholders } \\
\text { - Assess relevance } \\
\text { - Determine policies }\end{array}$ \\
\hline
\end{tabular}

Figure 4 - The key stages of policymaking through energy system modelling, adapted from (Mirakyan \& De Guio, 2013)

Within this context, then, the use of multi-vector models in the second phase shown in Figure 4 will only be a constructive effort if, firstly, they are capable of appropriately representing the questions and formulations derived in the first phase, and secondly, if their outputs are able to be relevantly assessed in the real-world context in the third phase. This requires those performing the modelling to closely appreciate the needs of their audience, and to avoid "modelling for modelling's sake". 


\section{Energy system actors and the solution space}

The decision variables within an energy model might not correspond to a real-world latitude in possible policy, particularly within deregulated markets. Where a model may select a specific technology over another (on a least-cost basis), the actual energy system to which the policies should be applied may involve technology-neutral markets and procurement mechanisms influenced by, for example, perceptions of risk and short time horizons.

The approach of Britain's gas and electricity regulator, Ofgem, towards regulating the future energy system seeks to ensure that "... regulation is neutral between different technologies, systems and business models ... promoting a level playing field between entrants and existing companies, and between network reinforcement and alternative solutions" through "promoting competition and harnessing market-based mechanisms" (Ofgem, 2017). This creates a disconnect between modelling and policy, in that most models select technologies based on assumptions of projected costs, whereas policies define or help to shape markets which are in turn used to select technologies based on actual, competitively-set prices. As most markets have regulatory distortions, this disconnection can emerge strongly from the outcomes of modelling that does not represent those regulations appropriately.

Typical policies which may be enacted involve incentives, subsidies and taxation. In this respect, energy consumers and producers may be 'nudged' towards particular technology selection, but the timescales on which this may occur may be very different to that envisaged by the optimum trajectory within an energy model. For example, the 'Green Deal' policy introduced in the UK in 2012, intended to improve financing for homeowners to invest in energy efficiency improvements, was cancelled in 2015 due to achieving an uptake of only 20,000 homes, compared to a target of 130,000 for the first year alone. In contrast, the Energy Company Obligation (ECO), by placing a statutory requirement on energy companies, has improved 1.4 million homes over the same period (NAO, 2016). While most whole system models see investment in energy efficiencies such as wall insulation as a near-term priority (in keeping with the negative cost seen in Marginal Abatement Cost curves), in the UK 12 million homes that could be insulated still do not have any form of wall insulation (ibid.), despite this being seen as a key policy area since the 2003 Energy White Paper. In this respect, the failure of existing techno-economic models to represent any non-technical or economic constraints on technology investments may lead to a recommended trajectory which is not feasible when behavioural and political requirements are considered.

This also raises the issue of endogenisation - the selection of which of the many individual elements and states of an energy system should be represented explicitly in the model, which should be left to be implicit within approximations, and which should be defined exogenously. Each quantified element of a multi-vector model should hence either be an endogenous variable or an exogenous parameter. For example, any energy system model will have the demand for energy (usually disaggregated by sector) as a key driver - but should this be set as external to the model, or derived within the model via use of other model elements and parameters such as economic activity and population? In the former case, the values derived may be set by a more detailed and appropriate model, but the latter case allows for some amount of demand responsiveness to be included (i.e. where demand itself responds to the price and availability of energy services).

Aspects of real-world systems which are not endogenised in the model may be represented by constraints selected to represent a restricted solution space judged as characteristic of the system in question. For example, limits to the rate of deployment of a particular technology may represent supply chain limitations not directly characterised within the model. While this is seemingly useful in 
making results more credible, these constraints are subject to the judgement of the modeller and may restrict the scope and impact of, for example, potential policy interventions (such as, in the given case, actions taken to enhance the development of the same supply chain). Within optimisation models it is usually possible to determine which constraints are binding and non-binding. Where constraints are applied as an approximation to real-world systems, the results should be interpreted with this additional knowledge, and the solution space adjusted accordingly where constraints are found to be binding and directly affecting the solution.

\section{Representation of physical properties}

An energy system model may simply treat energy as a single commodity which may flow to/from different locations or via conversion processes, with no difference in properties between different carriers. In other words, there may be no separate treatment of the flow of energy via gas pipelines as compared to electricity cables, other than their being separate flows which may not be mixed without conversion. In reality, the physics of the two are significantly different, with electricity flows governed by Kirchhoff's voltage and current laws, and gas flows driven by pressure differences and influenced by fluid dynamics. Including these physical constraints may lead to significantly different results, but greatly increase the computation required, and for some carriers may cause the problem to become non-linear. This creates a key modelling decision of the correct balance between model tractability and the scope, scale and accuracy of results. Models which do not include physical constraints may be 'soft-linked' with those that do in order to verify the results, such as in (Chiodi et al., 2011) where results of an energy systems model and a power system-specific model are compared for Ireland and differences in results are found to be relatively small but not insignificant.

A popular modern choice of energy system analysis, the 'Energy Hub', is a formulation first formally proposed by Geidl and Andersson in 2005 (Geidl \& Andersson, 2007) as an extension of previous optimal power flow frameworks to systems with multiple energy vectors. The idea was originally targeted at small distributed resource systems but can be used, generically, in respect of any spatial multi-vector energy system. One of the key benefits is that the framework may be used either incorporating the full physical description of different energy carriers, or more simply as a transporttype model in which a number of influences on flows through lines or pipes are neglected. The technique has been adapted to studies at a wide variety of scales, from local, $\mathrm{kW}$-scale domestic conversion systems such as in (Ramirez Elizondo, 2013), through urban networks incorporating renewable energy sources such as in (Niemi, Mikkola, \& Lund, 2012), to national-scale modelling such as in (Robertson \& Galloway, 2017). As well as the approach's adaptability to different spatial and temporal scales, there is scope for a model to be developed in sophistication over its lifetime, permitting so-called 'agile' modelling to be performed where a system is built up from simple demonstrative cases through prototyping. This also allows the early capture of model inconsistencies or misrepresentations, avoiding a black-box type model where it is unknown if unexpected outputs are accurate representations of reality or merely artefacts of the modelling process (Pfenninger et al., 2018).

\section{Spatial and temporal resolution}

Energy system models frequently cover large scales (entire neighbourhoods, regions or nations), and substantial periods of time (such as being optimised over a typical year, or over multi-year horizons for decarbonisation). The requirement for tractability may imply the use of low spatial or temporal resolutions. This choice of granularity may also permit easier interpretation of the results, which may assist where a model is being used for exploration of a concept rather than, for example, analysing 
security or reliability in a robust manner. The availability of suitably granular data may also restrict the resolution of modelling. National whole system models, such as UK TIMES, are often singleregion models with coarse blocks of time. However, this risks disguising many of the key technological features underlying the generation, conversion and consumption of energy, as well as obscuring many of the contributory elements of uncertainty (Usher \& Strachan, 2012).

With respect to temporal granularity, seasonal variation in energy is a key element of systems at higher/lower latitudes, where the consumption and production of energy will vary across the year. For example, in northern countries a large proportion of energy consumption will occur during winter months where the demand for heating is greatest. This will also drive international flows of energy commodities and the extent to which their prices vary in time.

Diurnal variation is similarly important in energy demand, with great variation between day and night due to patterns of human activity. The structure and nature of many real-world energy system assets are driven not by mean demand, but by the peak, which may be several times greater than the average and occur only for a brief period each day. For example, the GB National Grid uses the winter demand peak to determine the level of electricity generation capacity required to meet demand within a given level of reliability (National Grid, 2016), recognising that this is driven by the rate of consumption at the key seasonal and diurnal point. An energy system model which considers only the bulk averaging of demand risks significantly underestimating the capacity and cost of the energy system required to deliver security.

Increasing shares of variable power generation increase the importance of accurate representation of the temporal scale. With the growth of renewable sources of energy, and changing proportions of centralised versus disaggregated production, uncertainties and errors caused by under-representation of key temporal gaps will be magnified. The impact of temporal aggregation of renewable energy production is addressed in, for example (Pfenninger, 2017). This also highlights that the modelling techniques used may also need to evolve alongside the energy system they are attempting to represent, as the nature of that system itself changes over time.

Appropriate spatial detail is necessary to reflect demand, the varying availability and cost of resources in different locations, and constraints on the transfer of energy between different locations. Very localised variations, e.g. the viability of different heating technologies within the domestic sector, might be represented through disaggregation of sectors and definition of suitable costs and constraints for each sub-sector. However, if the data to distinguish such variations are not available then the extra development, maintenance and computational effort in establishing quite high spatial or sectoral resolution cannot be justified.

\section{Data Collection and Infilling}

Rarely is all the data desired by the modeller to parameterise their model both available and of sufficient quality. The broader the scope of the model, the greater the chance the modeller must rely on, or compensate for, low quality or missing data. A number of approaches are available to deal with this:

- Infilling of data from existing datasets through statistical analysis, such as in (Hawker, Bukhsh, Gill, \& Bell, 2016) where de-trended climate data is used to synthesise wind power output for new locations; 
- Use of proxy datasets to infill data, such as in (Neri, Coscieme, Giannetti, \& Pulselli, 2018) where night-time lighting data is used to infill power generation time series;

- Expert elicitation, such as in (Usher \& Strachan, 2013) where expert beliefs are pooled to determine drivers of energy demand and resultant commodity prices;

- Synthesis of data using appropriate simulation models.

A particular area frequently of issue to energy modellers is that of determining energy service demands - datasets are often limited in scope or extent, partially due to the paucity of data collection efforts, but also due to commercial and privacy concerns. Simulation efforts in this area have improved considerably in recent years, including for example, (Flett \& Kelly, 2016) where a model encompassing both building thermal properties and stochastic occupant behaviour is used to derive per-building energy service demand time series. Again, such models must be used with assumptions being fed through to the analysis of result sets, in order to avoid misinterpretation of outcomes based on hidden variables that have not been explicitly handled as part of the modelling exercise.

\section{RECOMMENDATIONS FOR FUTURE MODEL DESIGN}

\section{Choice of modelling framework and objective}

While beyond the scope of this review, the above discussion highlights the need for the formal specification of the analytical method used in an energy system model to be useful. For an optimisation model, this means that the objective function should be selected as representative of the dynamics of the system, e.g. a least-cost minimisation being used where an overarching economic driver exists or there is an actor who has the global purview to enact policies across that domain. This is, however, not restrictive, and least-cost optimisation can highlight failures in the regulatory environment where it can be shown that existing markets deviate from theoretically optimal outcomes. Robust and stochastic methods are of great use in determining the rigour of result sets (Panciatici et al., 2014), and avoid 'penny-switching' analyses which may result in singular outputs only representing very local solutions that represent perfect knowledge and decision making on the part of the modelled actors (Scott, Daly, Barrett, \& Strachan, 2016). A broader assessment of modelling objectives is given in (Mancarella et al., 2016).

\section{Auditing of inputs}

Models containing a large number of parameters may take a very long time to develop. Within this development time we might expect the state of knowledge for those parameters to change. For the sake of pragmatism, this may lead to a 'model freeze' where the model is based on the state of knowledge at a particular point in time rather than being continuously updated, meaning that the results may not correctly reflect current understanding. Alternatively, if a model is continuously updated with new information the state of knowledge encapsulated in different parts of the model may be inconsistent leading to, for example, incorrect selections between different technologies.

If the outputs of models are to be combined - either through joint modelling work or for assembling a policy evidence base - then the input assumptions should be consistent between those models.

A multi-vector model may contain a mixture of extant/mature technologies and relatively immature or speculative technologies. In this case, the costs and technological parameters of the former may be well understood and documented. However, parameters for the latter may be based on expert 
knowledge or simple conjecture that lacks robust justification or a clear 'audit trail'. If the two forms of inputs are directly compared without any allowance for this discrepancy in certainty, then the results may be more of a reflection of the level of optimism/pessimism of the projected technology than a robust analysis of relative futures. In (DECC, 2012), for example, this leads to a more favourable economic picture of relatively untested technologies such as heat pumps and thermal storage, compared to established business-as-usual cases, than might be evident if a range of costs were considered. Studies such as (Jalil-Vega \& Hawkes, 2017) improve upon this by considering a range of costs for competing technologies, based on a broad literature review though the chosen range of costs is still subject to judgment.

Sensitivity to judgments on model inputs is of particular importance when used in a context of decision making under uncertainty using approaches such as least regret where the extreme values of the range will define the regret values in use.

The requirements for consistency of inputs between modelling efforts, and the need for their robustness to be ensured through auditing, highlights the potential role of independent data curators (see inset). The task of data curation often has little value in terms of modelling innovation, so may be overlooked by academics (Mancarella et al., 2016).

\section{Coordination of models and modelling results}

The complexity of established models (both in terms of analytical frameworks and datasets) means that their evolution may occur in isolation, with little dissemination and collaboration between different mature modelling efforts. Some modelling communities (such as those using the TIMES framework, coordinated by ETSAP) are very active in collaborating between national models for mutual benefit, but often the funding incentives in research are aligned in a way that does not facilitate collaboration, and modelling efforts are often seen as being in competition with one another. This highlights the need for funding to set clear incentives for collaboration across institutions and disciplines.

There has been significant development in the open sourcing of energy models, both in modelling frameworks and input datasets. Efforts in this domain have suffered from issues around difficulties in determining data holders' rights as well as commercial sensitivities and the motivation to keep modelling closed for commercial benefit (Pfenninger et al., 2018). The gain to the modelling community, and those that seek to make use of the outputs, in avoiding sparsely documented, poorly maintained 'black box' models is clear but requires significant motivation to be achieved. Many energy system models have been developed within universities where there are, in general, only weak incentives in respect of documentation and maintenance of software and data. As a consequence, adequate resources are rarely sought or made available for them. It should also be highlighted that while the openness of models might support their rigour, it is not a direct replacement for peer review. However, the academic peer review process typically only reviews the methodologies and outputs of models and not their implementation in respect of coding and data sourcing.

As discussed in the above section on scenario selection and reduction, there may be a large range of results which must be presented to stakeholders - who may be experts in certain aspects of the modelling scope, but not others. Presentation of complex and multi-layered datasets may benefit from the use of interactive visualisation, where stakeholders can question and investigate result datasets alongside the modeller, such as in the use of interactive Sankey diagrams illustrating energy flows (Riehmann, Hanfler, \& Froehlich, 2005). 


\section{Quality Assurance and external validation}

In addition to defining the purpose and structure of a model, an additional required quality is that of assuring the model - that is, that it has been scoped, designed, validated, verified and documented. While such QA processes may not be explicitly defined or standardised for energy system modelling, there exist guidelines (such as the UK Government's Aqua Book (HM Treasury, 2015)) which define best practice for generating analytical evidence intended to contribute to public policy. A key discriminator which is often absent from academic modelling exercises is that of accountability - that a model must be signed off throughout its life cycle by someone of sufficient understanding and seniority to actively indicate that it is fit for purpose. This, in turn, returns to the conceptual requirement that the model design is appropriate to the questions it is intended to answer. In the academic space, the absence of prerequisite accountability is no barrier to abstract, conceptual modelling. However, it is perhaps prudent to always act as though such accountability is in place.

Simulation models are often relied upon to ensure the safe and reliable operation of engineering systems. It is essential that such a model's quality is validated and its parameters calibrated. This has been well-developed in specific domains such as building simulation (Coakley, Raftery, \& Keane, 2014), but many energy system models used, for example, to simulate the operation of markets or inform policy, are very difficult to validate. This therefore requires a high degree of awareness of a model's limitations on the part of its users but also, critically, an openness on the part of its developers in respect of the model design choices, the assumptions made and the sources of data. To aid a non-expert user, documentation of such features should be as accessible as possible taking the form, perhaps, of a high-level model description and 'assumptions catalogue' accompanied by greater technical detail documented separately that would allow an independent expert to reproduce the approach and test the results.

\section{The Role of Independent Data Curation}

Energy-based technologies evolve on a day-to-day basis. Immature technologies grow in maturity with each installation and manufacturing development through prototyping, demonstration and commercial deployment, generally in many different countries. Commercial viability is often enhanced by modelling work itself generating additional data to be used in further analysis. This makes it very difficult for any individual modelling research group to keep track of the most up-todate input parameters for their models, and also ensures that different models will generally have incompatible result sets for the purpose of joint analysis or soft-linking efforts. This means that there is great value in centrally-managed repositories of data upon which multiple research efforts may draw. However, such a repository needs to be sufficiently independent from the sources of data to objectively compare them. Data which may form part of the evidence base should be assessed prior to inclusion in any centralised dataset as there should be some sense checking of the rigour of proposed evidence, as well as filtering of data according to the reliability and independence of the source. While there is often a dependency on commercial actors for data, care must be taken to avoid being influenced by lobbying or optimism bias - see for example (HM Treasury, 2013).

Many individual repositories of data (such as from specific research projects) are decommissioned at the end of a funding lifecycle, making important references unavailable. While many funders of academic research now have stipulations around long-term data curation, this generally does not apply to commercial work, which generates a large proportion of data relevant to energy system analyses. 
An independent data curator can ensure that a combined data source outlives any individual hosting effort, effectively audit and review proposed material, and act as a central reference for consistency between individual modelling teams, reducing the need for duplication of effort and ensuring that modelling outputs are policy-relevant.

\section{CONCLUSIONS}

The growing diaspora of multi-vector energy system models means that there are a large number of analyses available to policymakers and commercial bodies aiming to make decisions about the direction of local, national and international energy systems, particularly within the context of national and subnational emissions targets and an increasing focus on energy system reliability and security. However, the tendency of such models to be both complex in formulation and complicated in having high dimensionality and numbers of input parameters means that, firstly, models are often not appropriately designed for the actual application of their outputs to real-world decisions and, secondly, that inputs may be poorly assessed and incorporated. Additionally, the decision/policymakers who may be using outputs from the models may have very little knowledge or understanding of the inputs and modelling assumptions in use.

Good practice in the conduct of 'useful' energy modelling should, as far as possible, do the following:

- analyse a timely and interesting element of the real-world flow of energy;

- assign an appropriate level of detail (spatial, temporal, physical) which allows the results to be meaningfully representative of the real-world system being analysed, and be open about the modelling choices made;

- either incorporate uncertainty directly into the model via robust or stochastic methods, e.g. through analysis of a range of scenarios, or interpret results within the context of assessed analytical and data uncertainties;

- produce results which may be usefully incorporated into a policymaking process or system design decision which is emerging or ongoing and, where possible, involve such stakeholders early in the modelling process;

- incorporate the best state of knowledge from multiple sources (academic, governmental, commercial), acknowledging assumptions, documenting sources and giving advice on uncertainties associated with those sources;

- make use of the full range of academic disciplines with useful input to the modelling, including practical insights from, for example, engineering and social sciences alongside theoretical advances in mathematics and operational research;

- where possible, contribute to the general energy modelling community through comparison of models and openness in data and methods, and incorporate lessons and data from other models to reflect best practice. Data processing tools, made by experts in the specific data processing area, can implement robust methodologies and use best sources of data, and be reusable for different modelling purposes. Being open, they would be available for outside inspection and development. 
It would be unreasonable to expect any single modeller of multiple energy vectors to be familiar with all of the technical and economic parameters within their model's scope. Modellers must therefore be able to turn to authoritative, published sources of combined and audited information in order to ensure that their work is rigorous and consistent with other work being conducted within academic, commercial and governmental research contexts. Commercial and other sensitivities may frequently prevent direct collaboration or openness in relevant datasets. These issues all point towards the important role that an active independent data curator can play. This also enables potential interdisciplinary collaboration with such curation acting as a common reference point for modellers and researchers through which they can become aware of each other's work and the ways in which future work may take advantage of knowledge covering multiple domains.

\section{Funding Information}

This work was undertaken within the UK Energy Research Centre (UKERC) Phase 3, supported by the UK Research Councils under Natural Environment Research Council award NE/ G007748/1 and EPSRC Grant No. EP/L024756/1.

\section{References}

Allan, G., Gilmartin, M., McGregor, P. G., Swales, J. K., \& Turner, K. (2009). Modelling the economy-wide rebound effect. In Energy efficiency and sustainable consumption (pp. 69-98). London, England: Palgrave Macmillan. https://doi.org/10.1057/9780230583108_4

Allan, G., Hanley, N., McGregor, P., Swales, K., \& Turner, K. (2007). The impact of increased efficiency in the industrial use of energy: A computable general equilibrium analysis for the United Kingdom. Energy Economics, 29 (4), 779-798. https://doi.org/10.1016/j.eneco.2006.12.006

Box, G. E. P. (1979). Robustness in the strategy of scientific model building. In Robustness in statistics (pp.201-236). Elsevier. https://doi.org/10.1016/B978-0-12-438150-6.50018-2

Bukhsh, W., Bell, K., Vergnol, A., Weynants, A., \& Sprooten, J. (2018). Enhanced, risk-based system development process: a case study from the Belgian transmission network. In 2018 power systems computation conference (PSCC)（pp. 1-9). IEEE. https://doi.org/10.23919/PSCC.2018.8442756

Chiodi, A., Deane, J. P., Gargiulo, M., \& Ó'Gallachóir, B. P. (2011). Modelling electricity generation Comparing results: From a power systems model and an energy systems model. In 30th international energyworkshop. https://web.stanford.edu/group/emf-research/newemf.stanford.edu/files/docs/323/Chiodi_Alessandro.pdf

Coakley, D., Raftery, P., \& Keane, M. (2014). A review of methods to match building energy simulationmodels to measured data. Renewable and Sustainable Energy Reviews, 37, 123-141.

https://doi.org/10.1016/j.rser.2014.05.007

DECC. (2012). RHI phase II - Technology assumptions.

https://www.gov.uk/government/publications/renewable-heat-incentive-phase-2-technology-assumptions

DEFRA \& Department for Transport. (2017). UK plan for tackling roadside nitrogen dioxide concentrations. https://www.gov.uk/government/publications/air-quality-plan-for-nitrogen-dioxide-no2-in-uk-2017

Department of Energy and Climate Change. (2011). Evaluation and learning from the 2050 public engagement programme $\cdot$ https://www.gov.uk/guidance/2050-pathways-analysis

E3Mlab of ICCS/NTUA. (2008). PRIMES model. Retrieved from http://www.e3mlab.ntua.gr/manuals/The_PRIMES_MODEL_2008.pdf 
Epe, A., Küchler, C., Römisch, W., Vigerske, S., Wagner, H.-J., Weber, C., \& Woll, O. (2009). Optimization of dispersed energy supply - Stochastic programming with recombining scenario trees (pp. 347-364). Berlin, Heidelberg: Springer. https://doi.org/10.1007/978-3-540-88965-6_15

TSAP. (2008). A comparison of the TIMES and MARKAL models. International Energy Agency. Retrieved from http://www.iea-etsap.org/web/TOOLS/TIMESVsMARKAL.pdf

Flett, G., \& Kelly, N. (2016). An occupant-differentiated, higher-order Markov chain method for prediction of domestic occupancy. Energy and Buildings, 125, 219-230. https://doi.org/10.1016/j.enbuild.2016.05.015

Geidl, M., \& Andersson, G. (2007). Optimal power flow of multiple energy carriers. IEEE Transactions on Power Systems, 22 (1), 145-155. https://doi.org/10.1109/TPWRS.2006.888988

Growe-Kuska, N., Heitsch, H., \& Romisch, W. (2003). Scenario reduction and scenario tree construction for power management problems. In 2003 IEEE Bologna power tech conference proceedings, vol. 3, pp. 152-158. https://doi.org/10.1109/PTC.2003.1304379

Hall, L. M. H., \& Buckley, A. R. (2016). A review of energy systems models in the UK: Prevalent usage and categorisation. Applied Energy, 169, 607-628. https://doi.org/10.1016/j.apenergy.2016.02.044

Hawker, G. S. (2018). Spatial and temporal disaggregation of whole system energy models through exemplar local multi-carrier networks. (PhD Thesis). University of Strathclyde.

Hawker, G. S., Bukhsh, W. A., Gill, S., \& Bell, K. R. W. (2016). Synthesis of wind time series for network adequacy assessment. In 19th power systems computation conference, PSCC 2016.

https://doi.org/10.1109/PSCC.2016.7540975

Heaps, C. G. (2016). Long-range energy alternatives planning (LEAP) system. Retrieved from https://www.energycommunity.org

HM Treasury. (2013). The supplementary green book guidance on optimism bias (pp. 1-15). https://www.gov.uk/government/publications/green-book-supplementary-guidance-optimism-bias

HM Treasury. (2015). The aqua book. https://www.gov.uk/government/publications/the-aqua-book-guidanceon-producing-quality-analysis-for-government

Jaccard, M. (2006). Sustainable fossil fuels: The unusual suspect in the quest for clean and enduring energy. Natural Resources Forum, 30 (4), 331-333. https://doi.org/10.1111/j.1477-8947.2006.116_1.x

Jalil-Vega, F., \& Hawkes, A. D. (2017). Spatially resolved model for studying decarbonisation pathways for heat supply and infrastructure trade-offs. Applied Energy, 210, 1051-1072.

https://doi.org/10.1016/j.apenergy.2017.05.091

Karangelos, E., \& Wehenkel, L. (2016). Probabilistic reliability management approach and criteria for power system real-time operation. In 2016 Power systems computation conference (PSCC) (pp. 1-9). IEEE. https://doi.org/10.1109/PSCC.2016.7540871

Ma, T., \& Nakamori, Y. (2009). Modeling technological change in energy systems - From optimization to agent-based modeling. Energy, 34 (7), 873-879. https://doi.org/10.1016/j.energy.2009.03.005

Mancarella, P., Andersson, G., Peças-Lopes, J. A., \& Bell, K. R. W. (2016). Modelling of integrated multienergy systems: Drivers, requirements, and opportunities. 2016 Power Systems Computation Conference (PSCC), Genoa. https.//doi.org/10.1109/PSCC.2016.7541031 
Mirakyan, A., \& De Guio, R. (2013). Integrated energy planning in cities and territories: A review of methods and tools. Renewable and Sustainable Energy Reviews , 22 , 289-297.

https://doi.org/10.1016/j.rser.2013.01.033

Müller, B., Gardumi, F., \& Hülk, L. (2018). Comprehensive representation of models for energy system analyses: Insights from the energy modelling platform for Europe (EMP-E) 2017. Energy Strategy Reviews, 21, 82-87. https://doi.org/10.1016/j.esr.2018.03.006

National Audit Office. (2016). Green deal and energy company obligation (summary). Retrieved from https://www.nao.org.uk/wp-content/uploads/2016/04/Green-Deal-and-Energy-Company-ObligationSummary.pdf

National Grid. (2016). Winter Outlook Report 2016/17.

https://www.nationalgrideso.com/document/61776/download

Neri, L., Coscieme, L., Giannetti, B. F., \& Pulselli, F. M. (2018). Imputing missing data in non-renewable empower time series from night-time lights observations. Ecological Indicators, 84, 106-118. https://doi.org/10.1016/j.ecolind.2017.08.040

Niemi, R., Mikkola, J., \& Lund, P. D. (2012). Urban energy systems with smart multi-carrier energy networks and renewable energy generation. Renewable Energy, 48, 524-536.

https://doi.org/10.1016/j.renene.2012.05.017

Ofgem. (2017). Our strategy for regulating the future energy system. https://www.ofgem.gov.uk/publicationsand-updates/our-strategy-regulating-future-energy-system

O'Malley, M., Kroposki, B., Hannegan, B., Madsen, H., Andersson, M., William, D., ... Dent, C. (2016). Energy systems integration: Defining and describing the value proposition. International Institute for Energy Systems Integration . https://doi.org/10.2172/1257674

Panciatici, P., Campi, M. C., Garatti, S., Low, S. H., Molzahn, D. K., Sun, A. X., \& Wehenkel, L. (2014). Advanced optimization methods for power systems. In 2014 power systems computation conference. IEEE. https://doi.org/10.1109/PSCC.2014.7038504

Pfenninger, S. (2017). Dealing with multiple decades of hourly wind and PV time series in energy models:A comparison of methods to reduce time resolution and the planning implications of inter-annual variability. Applied Energy, 197, 1-13. https://doi.org/10.1016/j.apenergy.2017.03.051

Pfenninger, S., Hawkes, A., \& Keirstead, J. (2014). Energy systems modeling for twenty-first century energy challenges. Renewable and Sustainable Energy Reviews, 33, 74-86. https://doi.org/10.1016/j.rser.2014.02.003

Pfenninger, S., Hirth, L., Schlecht, I., Schmid, E., Wiese, F., Brown, T., ... Wingenbach, C. (2018). Opening the black box of energy modelling: Strategies and lessons learned. Energy Strategy Reviews, 19, 63-71.

https://doi.org/10.1016/j.esr.2017.12.002

Ramirez Elizondo, L. M. (2013). Optimal usage of multiple energy carriers in residential systems. PhD thesis, TU Delft. https://repository.tudelft.nl/islandora/object/uuid:3e2cb6d7-3ba2-4b45-af71-

2fa106b5d189/datastream/OBJ/download

Riehmann, P., Hanfler, M., \& Froehlich, B. (2005). Interactive Sankey diagrams. In IEEE symposium on information visualization, 2005. INFOVIS 2005. (pp. 233-240). IEEE.

https://doi.org/10.1109/INFVIS.2005.1532152

Robertson, E., \& Galloway, S. (2017). Multi energy vector modelling of a Scottish energy system: Transitions and technology implications. Proceedings of the Institution of Mechanical Engineers, Part A: Journal of Power and Energy, 231 (6), 580-589. https://doi.org/doi.org/10.1177/0957650917711820 
Scott, K., Daly, H., Barrett, J., \& Strachan, N. (2016). National climate policy implications of mitigating embodied energy system emissions. Climatic Change, 136 (2), 325-338. https://doi.org/10.1007/s10584-0161618-0

Stephan, C., \& Sullivan, J. (2004). An agent-based hydrogen vehicle/infrastructure model. In Proceedings of the 2004 congress on evolutionary computation (IEEE Cat. No.04TH8753) (pp. 1774-1779). IEEE.

https://doi.org/10.1109/CEC.2004.1331110

Strachan, N. (2011). UK energy policy ambition and UK energy modelling-fit for purpose? Energy Policy, 39 (3), 1037-1040. https://doi.org/10.1016/j.enpol.2011.01.015

Tashiro, T., Tamura, K., \& Yasuda, K. (2011). Modeling and optimal operation of distributed energy systems via dynamic programming. In 2011 IEEE international conference on systems, man, and cybernetics (pp. 808813). IEEE. https://doi.org/10.1109/ICSMC.2011.6083752

Trutnevyte, E., McDowall, W., Tomei, J., \& Keppo, I. (2016). Energy scenario choices: Insights from a retrospective review of UK energy futures. Renewable and Sustainable Energy Reviews, 55, 326-337. https://doi.org/10.1016/j.rser.2015.10.067

Usher, W., \& Strachan, N. (2012). Critical mid-term uncertainties in long-term decarbonisation pathways. Energy Policy, 41, 433-444. https://doi.org/10.1016/j.enpol.2011.11.004

Usher, W., \& Strachan, N. (2013). An expert elicitation of climate, energy and economic uncertainties. Energy Policy , 61 , 811-821. https://doi.org/10.1016/j.enpol.2013.06.110

Van Beeck, N. (1999). Classification of energy models: FEW 777. https://doi.org/10.1.1.43.8055

van Vuuren, D. P., Hoogwijk, M., Barker, T., Riahi, K., Boeters, S., Chateau, J., ... Kram, T. (2009).

Comparison of top-down and bottom-up estimates of sectoral and regional greenhouse gas emission reduction potentials. Energy Policy, 37 (12), 5125-5139. https://doi.org/10.1016/j.enpol.2009.07.024

Wickart, M., \& Madlener, R. (2007). Optimal technology choice and investment timing: A stochastic model of industrial cogeneration vs. heat-only production. Energy Economics, 29 (4), 934-952.

https://doi.org/10.1016/j.eneco.2006.12.003

Zhou, Z., Zhang, J., Liu, P., Li, Z., Georgiadis, M. C., \& Pistikopoulos, E. N. (2013). A two-stage stochastic programming model for the optimal design of distributed energy systems. Applied Energy, 103, $135-144$. https://doi.org/10.1016/j.apenergy.2012.09.019 